$$
\frac{k \lambda-(k-1)}{(k-1) \lambda-(k-2)}=\frac{k^{\prime} \lambda-\left(k^{\prime}-1\right)}{\left(k^{\prime}-1\right) \lambda-\left(k^{\prime}-2\right)}
$$

the net result of which is $\left(k-k^{\prime}\right)(\lambda-1)^{2}=0$. Since $0 \leqq k<k^{\prime} \leqq n$, and the characteristic $p$ of $F$ is larger than $n, k-k^{\prime} \neq \equiv(p)$ and so $(\lambda-1)^{2}$ $=0$ results. Hence $\lambda=1$ and the theorem is proved.

We point out that the argument used above also works, up to a point in a Banach algebra. The conclusion one can reach is that the spectrum of $a b^{-1} a^{-1} b$ is invariant under the transformation $(2 \lambda-1) / \lambda$.

\title{
BIBLIOGRAPHY
}

1. C. Putnam and A. Wintner, On the spectra of commutators, Proc. Amer. Math. Soc. vol. 9 (1958) pp. 360-362.

Cornell University

\section{SOME ORTHOGONAL FUNCTIONS CONNECTED WITH POLYNOMIAL IDENTITIES}

J. B. ROBERTS

If $P(x)$ is an arbitrary polynomial of sufficiently small degree then there are various ways of choosing an integer $N$ and coefficients $c_{j}$ such that

$$
\sum_{j=0}^{N} c_{j} P(x+j)=0
$$

for all $x$. In each of $[3 ; 4]$ such an identity is proved. The present paper is devoted to a discussion of a connection between the coefficients in these identities and certain classes of orthonormal functions.

1. A polynomial identity and the Walsh functions. In [4] we proved the identity

$$
\sum_{n=0}^{b_{1}^{k+1}-1} \omega^{v_{b}(n)} P(x+n)=0,
$$

where $b$ and $k$ are positive integers, $v_{b}(n)$ is the sum of the coefficients

Presented to the Society, August 30, 1957 under the title Some classes of orthogonal functions; received by the editors December 16, 1957. 
of $n$ in base $b, \omega$ is an arbitrary $b$ th root of unity other than 1 , and $P(x)$ is any polynomial of degree $\leqq k$. For uniformity in notation with [1] we take $\omega=e^{2 \pi i / b}$.

We now define, for each $k$, a function of $x$ of period 1 whose values are precisely these coefficients on the successive intervals of $[0,1)$ which are of length $1 / b^{k+1}$.

DEFINITION 1.

$$
\begin{aligned}
w_{n}(x) & =\omega^{v b\left(\left[b^{n+1} x\right]\right)}, \quad 0 \leqq x<1 ; \\
w_{n}(x+1) & =w_{n}(x) \text { for all } x
\end{aligned}
$$$$
n \geqq 0
$$

Note that these functions depend on $b$. We shall not make this explicit in our notation as $b$ is to remain fixed.

Our first theorem states that $w_{n}(x)$ is the product of the first $n+1$ Rademacher functions of order $b$ (see [1]). The Rademacher functions of order $b$ are defined as follows.

$$
\begin{aligned}
r_{0}(x) & =\omega^{[b x]}, & 0 \leqq x \leqq 1 ; \\
r_{n}(x+1) & =r_{n}(x)=r_{0}\left(b^{n} x\right), & n \geqq 0 .
\end{aligned}
$$

THEOREM 1.

$$
w_{n}(x)=\prod_{j=0}^{n} r_{j}(x) .
$$

Proof. Suppose first that $0 \leqq x \leqq 1$. Then the coefficient of $b^{i}$ in the base $b$ expansion of $\left[b^{n+1} x\right]$ is congruent modulo $b$ to

Hence

$$
\left[\left[b^{n+1} x\right] / b^{j}\right]=\left[b^{n-j+1} x\right] .
$$

$$
v_{b}\left(\left[b^{n+1} x\right]\right) \equiv\left[b^{n+1} x\right]+\left[b^{n} x\right]+\cdots+[b x](\bmod b) .
$$

Therefore

$$
\begin{aligned}
\prod_{j=0}^{n} r_{j}(x) & =r_{0}(x) r_{0}(b x) \cdots \cdot r_{0}\left(b^{n} x\right) \\
& =r_{0}(x) r_{0}(b x-[b x]) \cdots r_{0}\left(b^{n} x-\left[b^{n} x\right]\right) \\
& =\omega^{[b x]} \omega^{\left[b^{2} x-b[b x]\right]} \cdots \omega^{\left[b^{n+1} x-b\left[b^{n} x\right]\right]} \\
& =\omega^{[b x]} \omega^{\left[b^{2} x\right]} \cdots \omega^{\left[b^{n+1} x\right]}=\omega^{v b\left(\left[b^{n+1} x\right]\right)}=w_{n}(x) .
\end{aligned}
$$

The theorem follows from periodicity.

The Walsh functions of order $b$ (see [1]) are defined by

$$
\begin{aligned}
& \psi_{0}(x)=1 \\
& \psi_{n}(x)=r_{n_{1}}^{a_{1}}(x) \cdots r_{n_{k}}^{a_{k}}(x)
\end{aligned}
$$


where the base $b$ expansion of $n$ is $a_{1} b^{n_{1}}+\ldots+a_{k} b^{n k}$. Hence from Theorem 1 we conclude that the sequence $\left\{w_{n}(x)\right\}$ is an orthonormal set since each $w_{n}(x)$ is a Walsh function. We also note that $\left\{w_{n}(x)\right\}$ is a lacunary subsequence of $\left\{\psi_{n}(x)\right\}$.

2. A similar situation with another polynomial identity. In [3] we proved the identity

$$
\sum_{n=0}^{b k-1} u_{b}(n+1) P(x+n)=0
$$

where $P(x)$ is a polynomial of degree less than $k(b-1)$ and the coefficients $\iota_{b}(n+1)$ are given in (4) below.

$b_{j}(n)$ is the coefficient of $b^{j}$ in the base $b$ expansion of $n$;

$$
\begin{aligned}
v_{b}(n) & =\sum_{j=0}^{\infty} b_{j}(n) ; \\
u_{b}(n+1) & =(-1)^{v_{b}(n)} \prod_{j=0}^{\infty}\left(\begin{array}{c}
b-1 \\
b_{j}(n)
\end{array}\right) .
\end{aligned}
$$

An easy consequence of (4) (see [3]) is the identity

$$
u_{b}\left(n+m b^{k}\right)=u_{b}(n) u_{b}(m+1) \text { for } 1 \leqq n \leqq b^{k}, \quad m \geqq 0 .
$$

Using the coefficients of (3) we construct a sequence of functions in analogy with the $w_{n}(x)$.

DEFINITION 2.

$$
\begin{array}{rlrl}
t_{n}(x) & =u_{b}\left(1+\left[b^{n+1} x\right]\right), \quad 0 \leqq x<1 ; & \\
t_{n}(x+1) & =t_{n}(x) \text { for all } x & & n \geqq 0 .
\end{array}
$$

Note that when $b=2$ we have $t_{n}(x)=w_{n}(x)$. This case is discussed in [3].

The natural analogues of the $r_{n}(x)$ out of which one can build the $t_{n}(x)$ would seem to be the functions $s_{n}(x)$ defined next.

Definition 3.

$$
\begin{aligned}
s_{0}(x) & =u_{b}(1+[b x])=(-1)^{[b x]}\left(\begin{array}{c}
b-1 \\
{[b x]}
\end{array}\right), & 0 \leqq x<1 ; \\
s_{n}(x+1) & =s_{n}(x)=s_{0}\left(b^{n} x\right), & n \geqq 0 .
\end{aligned}
$$

THEOREM 2.

$$
t_{n}(x)=\prod_{j=0}^{n} s_{j}(x)
$$

Proof. For $n=0$ this is evident. Hence we suppose it is true for 
$n \leqq k$. Then for $0 \leqq x<1, r=b^{k+1} x-\left[b^{k+1} x\right]$ we have

$$
\begin{aligned}
\prod_{j=0}^{k+1} s_{j}(x) & =t_{k}(x) s_{k+1}(x)=u_{b}\left(1+\left[b^{k+1} x\right]\right) s_{0}\left(b^{k+1} x\right) \\
& =u_{b}\left(1+\left[b^{k+1} x\right]\right) s_{0}(r)=u_{b}\left(1+\left[b^{k+1} x\right]\right) u_{b}(1+[b r]) \\
& \stackrel{*}{=} u_{b}\left(1+[b r]+b\left[b^{k+1} x\right]\right)=u_{b}\left(1+\left[b^{k+2} x\right]\right)=t_{n+1}(x) .
\end{aligned}
$$

At the asterisk we used (5). The conclusion now follows from periodicity.

3. Further properties of the $s_{n}(x)$ and $t_{n}(x)$ functions. In this section we prove a theorem concerning the orthogonality of power products of the $s_{n}(x)$. This theorem includes the orthogonality of the $t_{n}(x)$. We first prove two lemmas.

Lemma 1. $s_{0}(x)=u_{b}(k-[(k-1) / b])$ for $(k-1) / b \leqq x<k / b$.

Proof. Write $k-1=[(k-1) / b] b+r, 0 \leqq r<b$. Then $x=[(k-1) / b]$ $+(r+s) / b, 0 \leqq s<1$. Therefore $s_{0}(x)=s_{0}([(k-1) / b]+(r+s) / b)$ $=s_{0}((r+s) / b)=u_{b}(1+[r+s])=u_{b}(1+r)=u_{b}(k-b[(k-1) / b])$.

CorollaRY 1. $s_{n}(x)=u_{b}(k-b[(k-1) / b])$ for $(k-1) / b^{n+1} \leqq x$ $<k / b^{n+1}$.

Proof. $s_{n}(x)=s_{0}\left(b^{n} x\right)=u_{b}(k-b[(k-1) / b])$ for $(k-1) / b \leqq b^{n} x<k / b$.

CoROllaRY 2. $s_{n}(x)$ is constant over $\left[(k-1) / b^{m}, k / b^{m}\right)$ when $m>n$. In fact, $s_{n}(x)=u_{b}\left(1+\left[(k-1) / b^{m-n-1}\right]-b\left[\left[(k-1) / b^{m-n-1}\right] / b\right]\right)$ on this interval.

Proof. Let $k-1=b^{m-n-1}\left[(k-1) / b^{m-n-1}\right]+r$ where $0 \leqq r<b^{m-n-1}$. Then $k / b^{m-n-1}=\left[(k-1) / b^{m-n-1}\right]+(r+1) / b^{m-n-1} \leqq\left[(k-1) / b^{m-n-1}\right]+1$. Therefore

$\left[(k-1) / b^{m-n-1}\right] / b^{n+1} \leqq(k-1) / b^{m}<k / b^{m} \leqq\left(\left[(k-1) / b^{m-n-1}\right]+1\right) / b^{n+1}$ and the conclusions follow from Corollary 1.

LEMMA 2. $s_{n}(x)=s_{n}\left(x+1 / b^{n}\right)$.

Proof. $s_{n}\left(x+1 / b^{n}\right)=s_{0}\left(b^{n} x+1\right)=s_{0}\left(b^{n} x\right)=s_{n}(x)$.

Theorem 3. Let $f(x)$ be a power product of the $s_{n}(x)$ in which at least one $s_{n}(x)$ has exponent unity. Then $\int_{0}^{1} f(x) d x=0$.

Proof. Let $f(x)=P(x) Q(x) s_{n}(x)$ where

$$
\begin{aligned}
& P(x)=s_{a_{1}}^{\alpha_{1}}(x) \cdots s_{a_{p}}^{\alpha_{p}}(x), \\
& Q(x)=s_{c_{1}}^{\gamma_{1}}(x) \cdots s_{c_{q}}^{\gamma_{q}}(x)
\end{aligned}
$$


and $a_{i}<m<c_{j}$ for $1 \leqq i \leqq p, 1 \leqq j \leqq q$. By Corollary 2 of Lemma 1 , $P(x)$ is constant over $\left[(k-1) / b^{m}, k / b^{m}\right)$. Denote this constant by $P_{k}$. By Lemma 2,

$$
\int_{(j-1) / b^{m+1} \cdot}^{i / b^{m+1}} Q(x) d x=\int_{0}^{1 / b^{m+1}} Q(x) d x \text { for all } j .
$$

Now

$$
\begin{aligned}
& \int_{0}^{1} P(x) Q(x) s_{m}(x) d x=\sum_{k=1}^{b^{m}} \int_{(k-1) / b^{m}}^{k / b^{m}} P(x) Q(x) s_{m}(x) d x \\
& =\sum_{k=1}^{b^{m}} P_{k} \int_{(k-1) / b^{m}}^{k / b^{m}} Q(x) s_{m}(x) d x=\sum_{k=1}^{b^{m}} P_{k} \sum_{j=b(k-1)+1}^{b k} \int_{(j-1) / b^{m+1} \cdot}^{j / b^{m+1}} Q(x) s_{m}(x) d x \\
& =\sum_{k=1}^{b^{m}} P_{k} \sum_{j=b(k-1)+1}^{b k} u_{b}(j-b[(j-1) / b]) \int_{(j-1) / b^{m+1} \cdot}^{j / b^{m+1}} Q(x) d x \\
& =\left(\int_{0}^{1 / b^{m+1}} Q(x) d x\right) \sum_{k=1}^{b^{m}} P_{k} \sum_{j=b(k-1)+1}^{b k} u_{b}(j-b[(j-1) / b]) \\
& =\left(\int_{0}^{1 / b^{m+1}} Q(x) d x\right) \sum_{k=1}^{b^{m}} P_{k} \sum_{n=1}^{b} u_{b}(n) .
\end{aligned}
$$

But $\sum_{n=1}^{b} u_{b}(n)=0$ by (3) with $P(x) \equiv 1$. This completes the proof.

COROLlary. The sequence $\left\{t_{n}(x)\right\}$ is orthogonal.

Unfortunately it is not always true that $\int_{0}^{1} P(x) Q(x) d x=0$ where $P(x) \neq Q(x)$ are as in the proof of Theorem 3. In fact, if $P(x)=s_{0}(x)$ and $Q(x)=s_{0}^{2}(x)$ then, for $b=3$,

$$
\begin{aligned}
\int_{0}^{1} P(x) Q(x) d x & =\int_{0}^{1} s_{0}^{3}(x) d x=\int_{0}^{1}(-1)^{3[3 x]}\left(\begin{array}{c}
2 \\
{[3 x]}
\end{array}\right)^{3} d x \\
& =1 / 3-8 / 3+1 / 3=-2 .
\end{aligned}
$$

Hence one cannot complete the functions $s_{n}(x)$ by the method used in obtaining the Walsh functions of order $b$ from the functions $r_{n}(x)$. Also since for a given $b$ all functions of the form $P(x) Q(x) s_{m}(x)$, as in the proof of Theorem 3, are symmetric or antisymmetric about $1 / 2$, according to whether $b$ is odd or even, this set is far from complete. It would be interesting to find the completion of this set of functions.

4. Normalization of the $t_{n}(x)$. In this section we normalize the $t_{n}(x)$. Our first step is to prove 
Lemma 3.

$$
\sum_{i=1}^{b k} u_{b}^{2}(i)=\left(\sum_{i=1}^{b} u_{b}^{2}(i)\right)^{k}
$$

Proof.

$$
\begin{aligned}
& \sum_{i=1}^{b k} u_{b}^{2}(i)=\sum_{j=1}^{b k-1} \sum_{i=(j-1) b+1}^{j b} u_{b}^{2}(i)=\sum_{j=1}^{b k-1} \sum_{q=1}^{b} u_{b}^{2}((j-1) b+q) \\
& \stackrel{*}{=} \sum_{j=1}^{b k-1} \sum_{q=1}^{b} u_{b}^{2}(q) u_{b}^{2}(j)=\left(\sum_{q=1}^{b} u_{b}^{2}(q)\right)\left(\sum_{j=1}^{b k-1} u_{b}^{2}(j)\right)
\end{aligned}
$$

and the conclusion follows by induction. At the asterisk we used (5).

LEMMA 4.

$$
\int_{0}^{1} t_{n}^{2}(x) d x=\left(\left(\begin{array}{c}
2(b-1) \\
b-1
\end{array}\right) / b\right)^{n+1}
$$

Proof.

$$
\begin{aligned}
\int_{0}^{1} t_{n}^{2}(x) d x & =\int_{0}^{1} u_{b}^{2}\left(1+\left[b^{n+1} x\right]\right) d x=\sum_{j=1}^{b^{n+1}} \int_{(j-1) / b^{n+1}}^{j / b^{n+1}} u_{b}^{2}(j) d x \\
& =\left(\sum_{i=1}^{b} u_{b}^{2}(i)\right)^{n+1} / b^{n+1}=\left(\frac{1}{b} \sum_{i=1}^{b} u_{b}^{2}(i)\right)^{n+1} \\
& =\left(\frac{1}{b} \sum_{j=0}^{b-1}\left(\begin{array}{c}
b-1 \\
j
\end{array}\right)^{2}\right)^{n+1} \stackrel{*}{=}\left(\left(\begin{array}{c}
2(b-1) \\
b-1
\end{array}\right) / b\right)^{n+1} .
\end{aligned}
$$

At the asterisk we used the binomial identity

$$
\sum_{j=0}^{n}\left(\begin{array}{l}
n \\
j
\end{array}\right)^{2}=\left(\begin{array}{c}
2 n \\
n
\end{array}\right)
$$

obtained by equating coefficients of $x^{n}$ in the identity $(1+x)^{2 n}$ $=(1+x)^{n}(1+x)^{n}$.

Lemma 4 and the results of $\S 3$ yield the

Corollary. The sequence

$$
\left\{\left(b /\left(\begin{array}{c}
2(b-1) \\
b-1
\end{array}\right)\right)^{(n+1) / 2} t_{n}(x)\right\}
$$

$n \geqq 0$, is an orthonormal sequence.

5. A final lemma. In [2, page 45] it is proved that if $\phi(x)$ is a func- 
tion of $L^{2}$, of period 1, satisfying $\phi(x)+\phi(x+1 / 2)=0$ then the sequence $\left\{\phi_{n}(x)\right\}, n \geqq 0$, where $\phi_{n}(x)=\phi\left(2^{n} x\right)$, is orthogonal. Essentially the same proof gives

LEMMA 5. If $\phi(x)$ is a function of $L^{2}$, of period 1, satisfying $\sum_{j=1}^{b} \phi(x+(j-1) / b)=0$ and if $\phi_{n}(x)=\phi\left(b^{n} x\right), n \geqq 0$, then the sequence $\left\{\phi_{n}(x)\right\}$ is orthogonal on $[0,1]$.

Proof. Let $m>n$. Then

$$
\begin{aligned}
\int_{0}^{1} \phi_{m}(t) \phi_{n}(t) d t & =\left(1 / b^{n}\right) \int_{0}^{b^{n}} \phi\left(b^{m-n} u\right) \phi(u) d u=\int_{0}^{1} \phi\left(b^{m-n} u\right) \phi(u) d u \\
& =\sum_{j=1}^{b} \int_{(j-1) / b}^{j / b} \phi\left(b^{m-n} u\right) \phi(u) d u \\
& =\sum_{j=1}^{b} \int_{0}^{1 / b} \phi\left(b^{m-n}(v+(j-1) / b) \phi(v+(j-1) / b) d v\right. \\
& =\sum_{j=1}^{b} \int_{0}^{1 / b} \phi\left(b^{m-n} v+(j-1) b^{m-n-1}\right) \phi(v+(j-1) / b) d v \\
& =\int_{0}^{1 / b} \phi\left(b^{m-n} v\right)\left(\sum_{j=1}^{b} \phi(v+(j-1) / b)\right) d v=0
\end{aligned}
$$

One can prove directly by use of this lemma that the functions $s_{n}(x)$ are orthogonal. One merely takes $\phi(t)=s_{0}(t)$. One needs only show that $\sum_{j=1}^{b} s_{0}(t+(j-1) / b)=0$ and this is not difficult.

\section{REFERENCES}

1. H. E. Chrestenson, A class of generalized Walsh functions, Pacific J. Math. vol. 5 (1955) pp. 17-31.

2. S. Kaczmarz and H. Steinhaus, Theorie der Orthogonalreihen, New York, 1951.

3. J. B. Roberts, A curious sequence of signs, Amer. Math. Monthly vol. 64 (1957) pp. 317-322.

4. - , A new proof of a theorem of Lehmer, Canad. J. Math. (1958) pp. 191-194.

WESLEYAN UNIVERSITY AND

Reed College 